\title{
Aristote et le dualisme de la fin dans Physique II
}

Nélio Gilberto Dos Santos

In this study, we propose a revision of the Aristotelian passage in Physics, II, 2, 194a27-194b9, concerning the argument which aims to establish the dependence of matter on finality in natural processes. Considering the discussion on the translation of 194a32-36, and highlighting the notion of dualism of the final cause, we shall see how Aristotle presents his natural teleology according to a dual process determined not only by its culmination in a new natural form, but also by its use in a specific function.

Dans le livre II de la Physique, Aristote révèle son approche méthodologique de la philosophie naturelle à la lumière de quatre causes. Il s'agit d'un texte assez important du chapitre 2, 194a 27 - 194b 9 traitant du rapport entre matière et finalité, qui nous permet de mieux comprendre sa téléologie naturelle. Il convient cependant de reconnaître, à la lecture des interprètes, que ce texte n'a pas encore recueillit tout l'intérêt qu'il mérite, probablement pour deux raisons : d'abord par une excessive attention accordée à l'aspect épistémologique au détriment de la considération ontologique du processus naturel luimême ; ensuite, du fait de la sous-estimation de la notion de dualisme de la fin.

Cette étude propose une interprétation de ce passage à partir d'une relecture d'un extrait: 194a 32-36, dans lequel Aristote avance, par analogie avec le processus technique, une compréhension duelle de la téléologie qui a pour but d'intégrer l'idée d'usage et de fonction en tant que finalité, notions que nous savons centrale dans l'étude de la nature. Pour ce faire nous commencerons par soulever quelques problèmes posés par la traduction de ce court extrait qui, comme nous le verrons, se situe au cœur de l'argumentation. ${ }^{1}$

\footnotetext{
${ }^{1}$ Suzanne Mansion, dans une étude consacrée à ce passage, considérait qu'il y avait en 194a 3236 "la clé de tout le morceau". Cf. S. Mansion, "Un passage obscur du deuxième livre de la Physique (ch. 2, 194a 27-b8)", in S. Mansion, Études Aristotéliciennes, recueil d'articles, avantpropos, bibliographie, indices par J. Follon, Louvain-la-Neuve, Ed. de l'Institut supérieur de philosophie, 1984, p. 361.
} 


\section{Quelques remarques sur la traduction}

Ce passage de Physique II, 2, 194a 32-36 a récemment été mis en lumière par des commentateurs à la suite de la thèse que D. Sedley a émise dans un article où il défend une lecture anthropocentrique de la téléologie de ce texte. ${ }^{2}$ En effet, les interprètes modernes ne se sont pas montrés insensibles à cette question, et ont proposés des traductions atténuant ce sens. Lisons avant tout ces quelques lignes dans le texte grec de l'édition de Ross, ainsi que dans sa traduction latine médiévale:

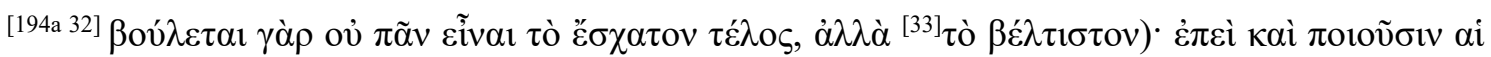

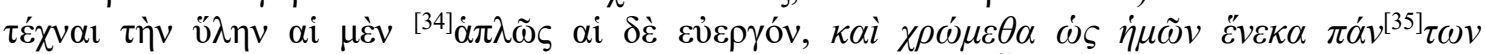

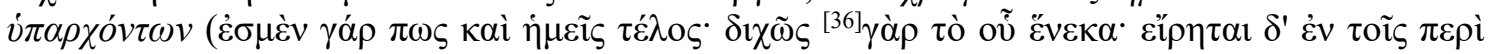

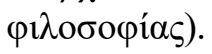

La Vetus en donne la traduction suivante :

vult enim non omne esse ultimum finem, sed optimum ; quoniam et faciunt artes materiam alie quidem simpliciter, alie autem operose, et utimur tamquam propter nos omnibus que sunt (sumus enim quodammodo et nos finis; dupliciter enim est id cuius causa fit, dictum est autem in his que sunt de philosophia). (Cf. Aristoteles Latinus, VII. 1. 2, Physica, pp. 53-54.)

La difficulté de comprendre l'idée figurant dans la phrase ci-dessus soulignée, où l'homme est considéré la fin de toutes choses, dans la perspective immanentiste de la téléologie d'Aristote, a conduit à une lecture du $\omega \varsigma$ de la ligne 194a 34 comme un contrefactuel. Ainsi, Hardie et Gaye, par exemple, traduisent:

(... For not every stage that is last claims to be an end, but only that which is best.) For the arts make their material (some simply make it, others make it serviceable), and we use everything as if it was there for our sake. (We also are in a sense an end. 'That for the sake of which' may be taken in two ways, as we said in our work On Philosophy.) (Cf. The complete works of Aristotle: The revised Oxford translation, N.J, Princeton University Press, p. 332).

Ce passage ainsi traduit impose une importante inflexion de sens. Aristote prendrait alors position contre l'idée anthropocentrique commune qu'il rapporterait sans la partager, car le contrefactuel "comme si" sous-entend qu'en réalité "nous" ne sommes pas une fin véritable. Cette lecture a été vivement critiquée par D. Sedley qui traduit lui-

\footnotetext{
${ }^{2}$ Cf. D. Sedley, “Is Aristotle's Teleology Anthropocentric?”, Phronesis, vol. 36, n. 2, 1991. Voir aussi: "Teleology, Aristotelian and Platonic", in G. Lennox et R. Bolton (éd.), Being, Nature and Life in Aristotle. Essays in Honor of A. Gotthelf, Cambridge, Cambridge University Press, 2010, pp. 5-29.
} 
même : "And we use it on the ground that everything exists for our sake". ${ }^{3}$ Le $\dot{\omega} \varsigma$ n'aurait donc plus la fonction d'introduire une opinion douteuse, voire erronée, il servirait plutôt à expliquer sur quelle base il est possible de légitimer l'usage des artefacts. ${ }^{4}$

Ce problème du sens de $\hat{\omega} \varsigma$ a été repris par M. Leunissen qui défend plutôt la traduction par le contrefactuel. Elle s'appuie sur la grammaire afin de montrer qu'il s'agit là d'un artifice permettant de prendre ses distances lorsqu'on fait allusion à l'opinion de quelqu'un autre. ${ }^{5}$ Le texte cité à l'appui, l'Anabase de Xénophon, IV, 2, 5-6, est clair. Lisons ce passage dans l'édition et la traduction de P. Masqueray :

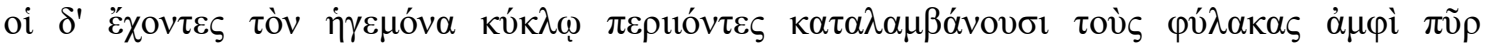

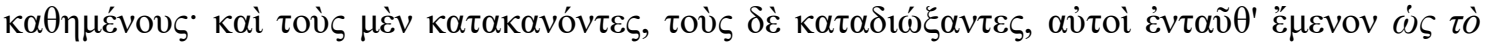

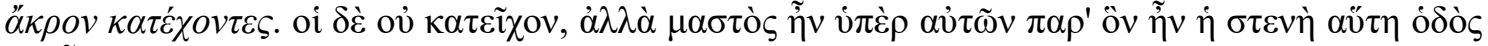

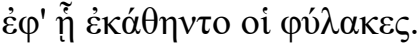

De leur côté ceux qui avec le guide s'avançaient par un détour circulaire tombent sur les gardes ennemis assis autour du feu; ils en tuent un certain nombre, poursuivent des autres et s'installèrent à leur tour en cet endroit, croyant être maîtres de la hauteur. Ils ne s'en étaient pas rendus maîtres : il y avait un mamelon au-dessus d'eux, le long duquel était l'étroit chemin où étaient assis les gardes (Xénophon, Anabase IV-VII, Les Belles Lettres, Paris, 1961, pp. 12-13).

Dans ce cas, en effet, le sens subjectif et contrefactuel du ós est vérifié. Par cet artifice, l'auteur signale la cause ou le fondement d'une attitude par cette opinion rapportée qui, d'après le contexte, est manifestement fausse. En effet, Xénophon se désolidarise de la pensée des guerriers qui se trouvaient dans l'illusion d'avoir pris le sommet. Il importe de noter, néanmoins, qu'en raison de l'évidence dans le passage la traduction du $\omega \varsigma$ par "croyant", dans la traduction citée, convient tout aussi bien qu'une traduction par "comme si", sans risque de tomber dans un contre-sens, ce qui n'est pas le

\footnotetext{
${ }^{3}$ Notons que la traduction est assez proche de celle de la Vetus citée plus haut, ainsi que de celle du commentaire de Ross : "(... which is absurd because not every final point but only that which is best is a final cause). Indeed, some arts make their matter and others make it workable, and we use their matter as existing for our own sake (for we are the end in one of the two senses of ' end')". Cf. Aristotle's Physics, a revised text with introduction and commentary by W. D. Ross, 2 vol., Oxford, Clarendon, 1936, vol. I, p. 351.

${ }^{4}$ Sedley précise: "Here the middle sentence is frequently translated 'And we use it as if everything existed for our sake. But the construction, $\omega \varsigma$ plus participle, does not have any such counterfactual implication, and this usual translation must merely reflect an interpretative prejudice”. Cf. D. Sedley, “Is Aristotle's Teleology Anthropocentric?”, cit., p. 189.

${ }^{5}$ Leunissen explique en note: " according to Rijksbaron et al. (2000, 100), constructions like these indicate a 'subjective reason' for which the narrator does not want to be held responsible (cf. X. $A n$ IV. 2.5). Aristotle thus make a concession to popular thought in claiming that we are in some sense (pôs should be read in a conciliatory way) an end, too." Cf. M. Leunissen., Explanation and teleology in Aristotle's science of nature, Cambridge, Cambridge University press, 2010, p. 39, n. 80 .
} 
cas dans Physique II, où le choix du traducteur peut déterminer des sens opposés. ${ }^{6}$ La justification de l'emploi du $\omega \varsigma$ pour exprimer la pensée d'autrui est, certes, vérifiée dans le texte de Physique II, mais rien ne semble prouver qu'Aristote la considère fausse. Par ailleurs, le sens contrefactuel est atténué chez Leunissen elle-même puisque, selon sa lecture, Aristote n'y refuse pas totalement ce finalisme, mais l'intègre dans ce qu'elle nomme une téléologie "secondaire". 7

Ainsi, la traduction défendue par Sedley : "on the ground that", est, sans aucun doute préférable en tant qu'elle garde la valeur d'indétermination présente dans $\omega \varsigma$, mais il importe également d'indiquer aussi que le sens contrefactuel, "as if", reste grammaticalement possible si le contexte l'accepte. ${ }^{8}$ Autrement dit, le sens contrefactuel doit être suffisamment évident pour qu'une formulation plus claire ne soit pas utilisée, comme ce serait le cas pour $̋ \sigma \pi \varepsilon \rho$ avec ö́v ou/et $\varepsilon \dot{i}^{9}{ }^{9}$ C'est donc le contexte, et non pas uniquement la nuance subjective apportée par $\omega \varsigma$, qui nous permet d'affirmer que l'idée ou la croyance ainsi rapportée est erronée, ou encore que l'auteur qui l'a décrite ne l'admet pas.

La deuxième remarque porte, cette fois-ci, sur l'objet du verbe $\chi \rho \alpha ́ o \mu \alpha 1$, dans la même phrase, 194a 34-35, précédant ஸ́s. On peut lire, par exemple, dans la traduction de Hardy et Gaye citée plus haut : "and we use everything as if it was there for our sake".

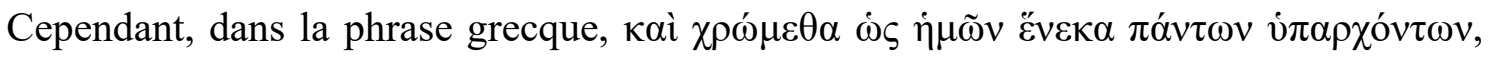

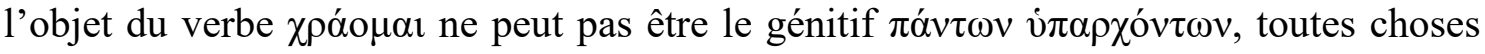

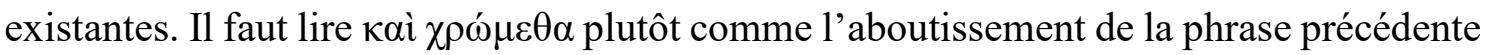

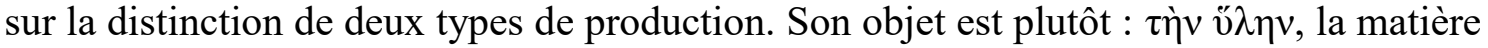
produite $\dot{\alpha} \pi \lambda \tilde{\omega} \varsigma$ et $\varepsilon \dot{\varepsilon} \rho \gamma o ́ v .{ }^{10}$ Ainsi, Aristote ne dit pas "nous utilisons toutes les choses qui existent", ce qui d'ailleurs n'aurait pas de sens. Il faudrait plutôt lire: "et nous l'utilisons, dans la pensée que toutes choses existent en vue de nous". Le $\dot{\omega} \varsigma$ après le

${ }^{6}$ On peut aussi voir une possibilité de contre-sens dans les différentes traductions françaises de Phys VI, 10, 240b 14-17 chez Carteron et Pellegrin.

${ }^{7}$ Cf. M. Leunissen, Explanation and teleology, cit., pp. 39- 40.

${ }^{8}$ Sur ce point il est intéressant de remarquer que ce texte de Physique II, même sans la traduction du $\omega \varsigma$ par un contrefactuel, est cité pour réfuter l'idée d'une téléologie anthropocentrique chez Aristote par M.-P. Lerner, Recherches sur la notion de finalité chez Aristote, Paris, PUF, 1969, p. 36.

${ }^{9}$ On peut retrouver ces formulations un peu plus loin : Phys II, 8, 198b 29-32 et 199b 19-22.

${ }^{10} \mathrm{Ce}$ sens de la matière comme produit peut être rapproché de celui donné dans $P o l \mathrm{I}, 8,1256 \mathrm{a}$

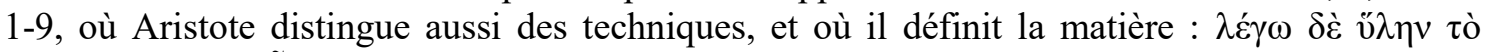

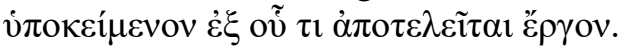


verbe, dans ce cas, n'est pas une complétive qui introduirait l'objet, il sert plutôt à nuancer

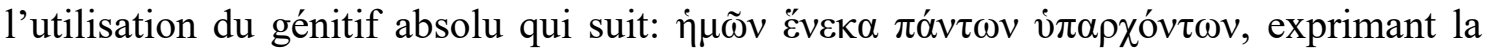
raison qui fonde l'action du verbe en question, $\chi \rho \alpha ́ o \mu \alpha \iota$ : nous nous servons de ces choseslà / ayant cette pensée.

Ces remarques permettent de préciser un autre point : à quoi Aristote se réfère-til en employant $\pi \alpha ́ v \tau \omega v$ dans cette phrase ? Il a été montré que ce n'est pas l'objet de $\chi \rho \omega ́ \mu \varepsilon \theta \alpha$, et le participe $v \pi \alpha \rho \chi o ́ v \tau \omega \nu$ ne doit pas être compris comme un adjectif: "toutes

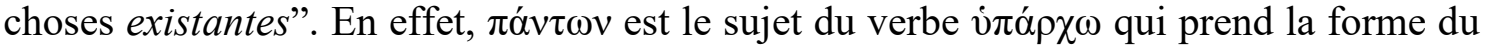
participe génitif, en raison du génitif absolu. Selon certains, la totalité à laquelle Aristote fait ici allusion serait simplement celle à laquelle se réfère le verbe $\chi \rho \omega ́ \mu \varepsilon \theta \alpha$, c'est-à-dire, la totalité de la matière produite $\dot{\alpha} \pi \lambda \tilde{\omega} \varsigma$ et $\varepsilon v ̉ \varepsilon \rho \gamma o ́ v$, ces choses que "nous utilisons". ${ }^{11}$ Le problème de cette lecture, qui, certes, rend le sens de la phrase moins problématique, est

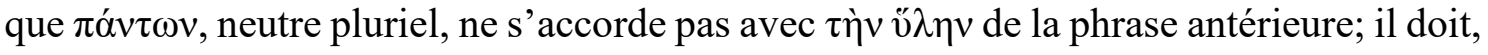
par conséquent, être lu d'une façon plus indéterminée comme "toutes choses" de façon générale, c'est-à-dire toutes les choses naturelles.

Il s'agit ainsi, par cette phrase au génitif absolu introduite par $\omega \varsigma$, de fonder l'usage technique dans une explication téléologique plus fondamentale dans laquelle l'homme est fin, non seulement en raison de l'intentionnalité de la production technique, mais en raison d'une vision téléologique plus large, où le monde physique est ordonné à l'usage humain. Le $\pi \alpha ́ v \tau \omega v$, dans ce texte, aurait alors le même sens que $\pi \alpha ́ v \tau \alpha$ du passage que l'on a rapproché, à juste titre, dans Politique I, 8, c'est-à-dire 'toutes choses du monde physique", celles que la nature destine à l'homme. ${ }^{12}$

\section{Le dualisme de la fin}

Après ces précisions sur la traduction, replaçons cet extrait dans son contexte, à savoir dans l'ensemble du passage 194a 27 - 194b 9 de Physique II, 2, qui forme, sans conteste, une unité d'argumentation autour de la relation entre matière et finalité.

${ }^{11}$ C'était la compréhension de Simplicius : in Aristotelis Physicorum libros quattuor priores commentaria, CAG, t. IX, ed. Hermannus Diels, Vol. IX, Berolini, G. Reimer, 1882, 304, 10-13. Et encore la traduction de Wicksteed et Cornford: The Physics, with an English translation by P. H. Wicksteed \& F. M. Cornford, 2 vol., London ; Cambridge, W. Heinemann ; Harvard University Press, 1980.

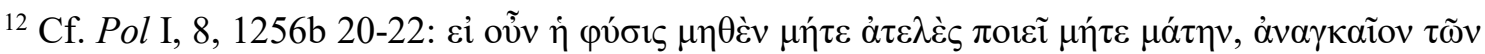

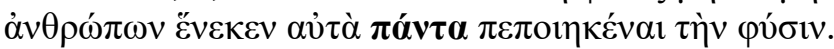


L'affirmation initiale est celle qu'Aristote cherchera à démontrer par la suite: l'étude de la finalité, ainsi que celle de la matière, relèvent de la même science, c'est-à-dire de la Physique. L'idée centrale, ici, est de défendre, face aux physiciens, l'idée qu'il ne peut y avoir de véritable connaissance de la matière sans référence à la cause finale. Penchonsnous sur ce passage :

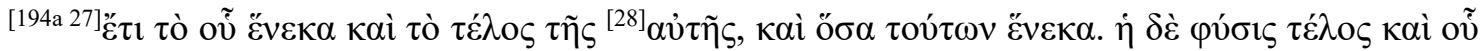

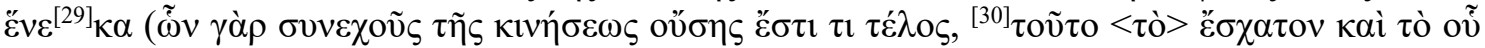

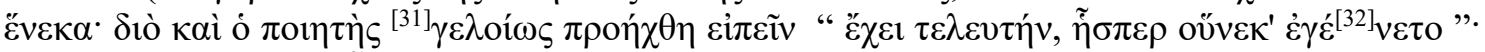

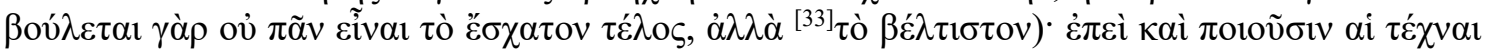

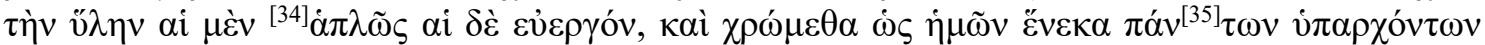

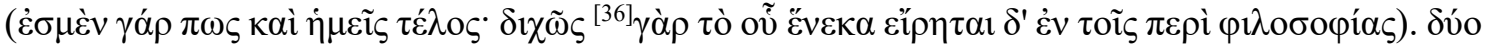

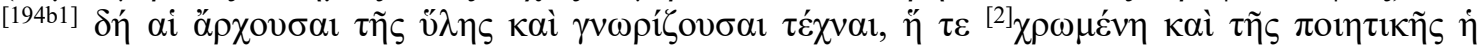

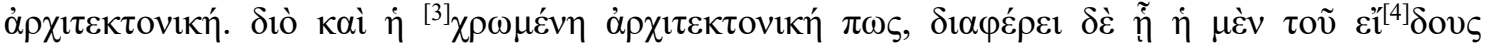

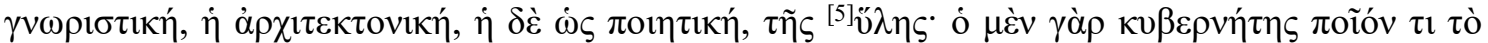

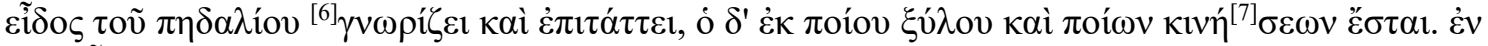

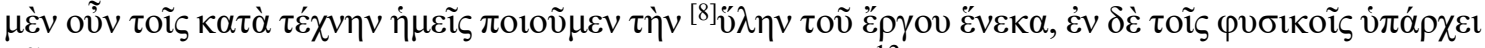

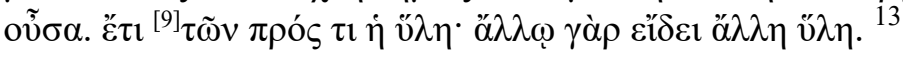

En outre, appartiennent à la même < science> le ce en vue de quoi, c'est-à-dire la fin, et tout ce qui est en vue de ces choses. La nature en effet est fin et ce en vue de quoi (en effet, les choses en mouvement continu ont une certaine fin qui est son terme et ce en vue de quoi). C'est pourquoi le poète laisse aussi échapper, de façon ridicule: "Il atteint l'accomplissement pour lequel il est né", car tout terme ne prétend pas être une fin, mais seulement le meilleur).

Puisque les techniques produisent leur matière, les unes d'une façon simple, les autres d'une façon adaptée à une fonction, et qui nous nous en servons dans l'idée que tout soit en vue de nous, (car, d'une certaine façon, nous aussi nous sommes fin, en effet, le ce en vue de quoi est double, comme il a été dit dans le traité Sur la Philosophie); par conséquent, il y a deux techniques qui commandent et qui connaissent la matière : les techniques de l'usage et celles qui ordonnent la technique de production.

C'est pourquoi les techniques de l'usage sont aussi, d'une certaine façon, ordonnatrices, la différence entre elles est que la technique de l'usage ordonne en tant qu'elle connaît la forme ; l'autre, en revanche, en tant que productrice, connaît la matière. En effet, tandis que le timonier connaît et prescrit la forme d'un gouvernail, l'autre connaît à partir de quel bois et de quels changements on peut le produire. En fait, dans les réalités techniques nous faisons la matière en vue de l'œuvre, mais, dans les réalités naturelles la matière est donnée. De plus, celle-ci fait partie des choses relatives, car, pour une forme différente, il faut une matière différente. ${ }^{14}$

Dans ce texte, Aristote commence par inscrire la finalité dans le mouvement de la nature dans son ensemble: les choses naturelles ont une orientation téléologique; les changements dans la matière ne sont pas dus au hasard, mais ils tendent vers le meilleur.

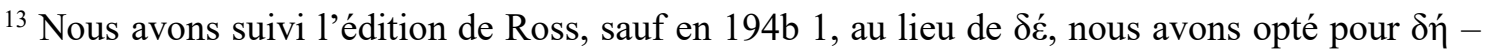
des manuscrits $\mathrm{IJ}^{1} \mathrm{~F}$, ce qui était déjà le choix dans l'édition de Bekker -, afin de marquer textuellement la dépendance évidente entre la première distinction dans le processus technique, et la seconde qui débute.

${ }^{14}$ Nous traduisons. 
Cette considération d'une structure téléologique fondamentale dans la nature sera expliquée par analogie avec le processus technique dans la suite du passage.

En effet, à la ligne 194a 33, Aristote entame une argumentation dans laquelle il précise la façon dont la matière est relative à la finalité dans l'art. Deux distinctions concernant les techniques ponctuent son raisonnement. Tout d'abord, en 194a 33-34, celle

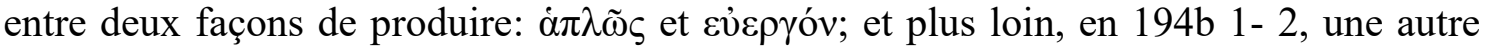
distinction en découle: celle entre la technique ordonnatrice de la production et la technique de l'usage. Ces deux distinctions ne se recouvrent pas. La dernière est assez commune sous la plume d'Aristote, dont le but ici est d'expliciter deux façons de connaître et de dominer la matière. Quant à la première distinction, $\dot{\alpha} \pi \lambda \tilde{\omega} \varsigma$ et $\varepsilon v ̉ \varepsilon \rho \gamma o ́ v$, il s'agit de deux types de production de la matière et, même si elle n'est pas absente des textes, elle ne revient plus sous ces termes. ${ }^{15}$

Cependant, bien comprendre cette distinction entre deux manières de produire est fondamental, parce que toute l'argumentation commence par là, et qu'en outre, c'est dans sa suite que la dualité de la fin est invoquée. Puisqu'on ne peut donner un sens précis à l'expression $\dot{\alpha} \pi \lambda \tilde{\omega} \varsigma$ sans faire référence à l'autre terme auquel elle s'oppose, il est

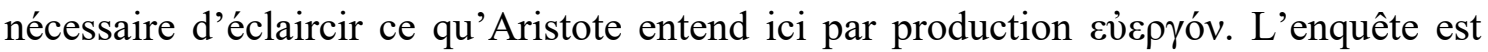
brève car il n'y a que deux occurrences de celle-ci. Tout d'abord il convient de remarquer qu'elles ne concernent plus le processus technique, mais plutôt celui de la nature : soit de l'inanimé dans Météorologiques; ou à propos des vivants dans Les Parties des Animaux. Dans ces deux occurrences, le sens est assez proche. Pour la première, il s'agit de qualifier un état particulier de l'air disposé à devenir de l'eau ${ }^{16}$; quant au second, il s'agit de la production naturelle d'un organe qui soit adapté et disposé à un usage postérieur.

Ce passage de Les Parties des Animaux est particulièrement éclairant. En II, 14, 659b 34 - 660a 11, il est, en effet, question de la constitution particulière de la langue et des lèvres humaines expliquées par rapport à leurs fonctions. Il s'agit d'une considération

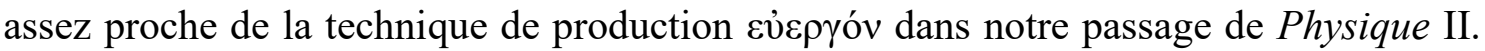

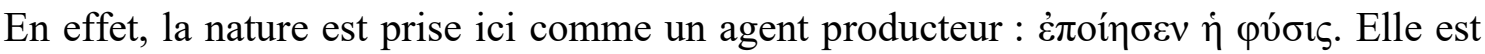

${ }^{15}$ Néanmoins, on peut la rapprocher du passage déjà cité de Pol I, 8, 1256a 1-9, dans lequel Aristote distingue une technique qui procure des instruments, et une autre qui fournit la matière.

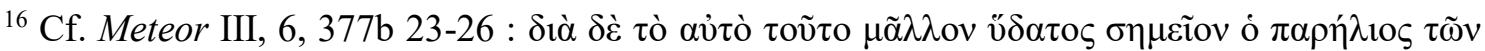

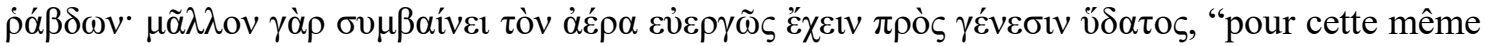
raison, le parhélie est davantage signe de la pluie que les raies, car il signifie davantage que l'air est dans un état propice à la formation de pluie" (Trad. J. Groisard : Aristote, Euvres Complètes, sous la direction de P. Pellegrin, Paris, Flammarion, 2014, p. 937). 
responsable du fait que les parties du corps, dans ce cas les lèvres et la langue humaines,

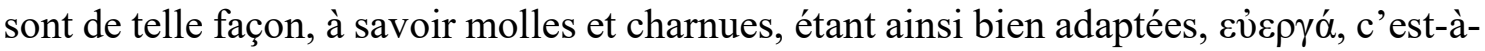

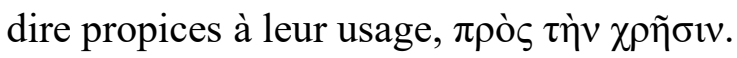

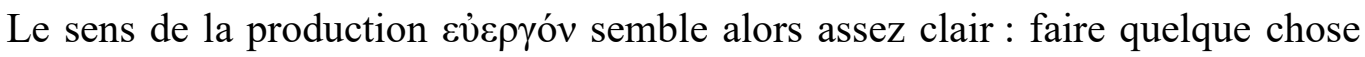
pour un usage spécifique, une production plus sophistiquée et précise à laquelle Physique II oppose la production $\dot{\alpha} \pi \lambda \tilde{\omega} \varsigma$. On peut alors en déduire que celle-ci, par contraste, est

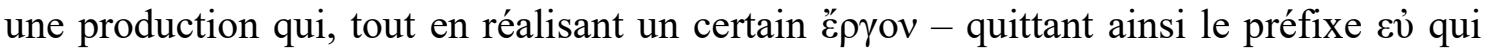
rajoute un sens d'aisance et de facilité -, aboutit à un produit qui n'a pas toute la finesse d'adaptation à une fonction spécifique, il s'agirait d'un produit plus primitif, moins élaboré. On peut penser, par exemple, à de la laine en tant que production $\dot{\alpha} \pi \lambda \tilde{\omega} \varsigma$, et à une

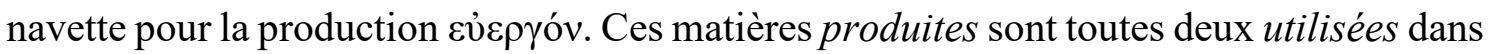
l'art du tissage. ${ }^{17}$

Cette distinction entre deux types de production dans Physique II souligne un élément important pour comprendre la relation entre matière et finalité. En effet, comme Aristote le rappelle à la fin du passage (194b 7-8), la production technique d'une matière est en vue de l'œuvre, है $p \gamma o v$. La technique vise un nouvel artefact matériel par une transformation donnant une détermination nouvelle à une matière donnée ; par exemple, la fin de la construction navale est le bateau. ${ }^{18}$ Mais le mot $̌$ है $\rho$ ov ne signifie pas seulement le produit achevé, la matière avec sa forme artificielle, ěpyov dit aussi fonction, c'est-àdire l'usage de l'artefact. ${ }^{19} \mathrm{C}$ 'est cette idée de Ěprov, non pas seulement en tant qu'il s'agit d'un composée hylémorphique achevé, d'une façon statique, mais plutôt en tant que fonction, c'est-à-dire dans sa dynamique propre dans un usage spécifique, qui intéresse Aristote et qui est mise en lumière dans la suite de notre passage de Physique II.

Aristote précise, en effet, que "nous", les utilisateurs des produits techniques, le tisserand par exemple, nous utilisons ce qui a été fait, soit $\dot{\alpha} \pi \lambda \tilde{\omega} \varsigma$, soit $\varepsilon v ̉ \varepsilon \rho \gamma o ́ v$, en nous considérant non pas comme des bénéficiaires accidentels, ou même comme une partie importante dans le processus de production, mais plutôt comme sa finalité même. La raison présentée se réfère à la pensée courante dans la phrase dont nous avons discuté la traduction: "dans l'idée que tout soit en vue de nous". La compréhension de la finalité

${ }^{17}$ Ce sont les exemples donnés par Aristote dans le passage cité antérieurement en note : Pol I, 8, 1256a 1-9.

${ }^{18}$ Cf. EN I. 1, 1094a 8-9.

${ }^{19}$ La fin de la production d'une scie est celle-ci et aussi son usage, ou fonction, ع́pyov: Phys II, 200a 9-10. 
technique, ici, n'est donc pas celle d'un simple destinataire qui utiliserait les artefacts selon sa pure subjectivité. Cet usage, pour être une véritable fin, et non pas un processus violent ou contre nature, doit être fondé, en quelque sorte, dans le processus naturel de la chose matérielle elle-même. En effet, chez Aristote, la technique n'est jamais montrée comme une pure ingérence humaine dans le devenir naturel, il s'agit plutôt d'une certaine continuation, d'une "imitation" et d'un "achèvement" de la nature. ${ }^{20}$

Cette phrase, revoyant sans doute à la pensée courante, est celle nuancée par $\dot{\omega} \zeta$ que nous avons traduit par "dans l'idée que". Aristote partage-t-il cette vision anthropocentrique? Si non, la suite doit nous le faire comprendre, mais, dans le cas contraire, pourquoi s'y référer en tant qu'opinion? Tout d'abord, il convient de noter, contrairement au sens contrefactuel du $\hat{\omega} \zeta$, que la suite du passage ne nie pas cette vue commune, elle montre plutôt qu'Aristote veut que sa téléologie en rende compte. En effet, deux

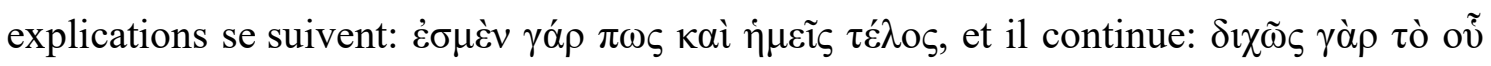

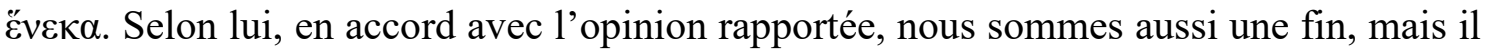
apporte une nuance: "d'une certaine façon", $\pi \omega \varsigma$. Quelle est alors cette manière particulière d'être fin ? Il faut avouer que rien n'indique un sens affaibli de la finalité, car son explication n'est pas une considération pour un cas spécifique, au contraire, il s'agit

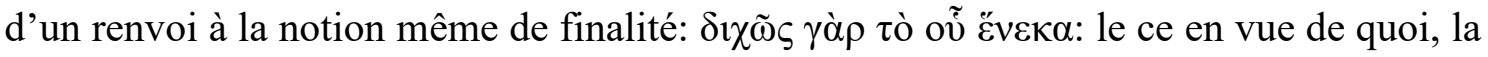
cause finale, est elle-même double.

Ainsi, qualifier l'homme de fin d'une certaine façon, montre la volonté de l'auteur de préciser, voire même de corriger, le sens du finalisme anthropocentrique de l'opinion courante. Cette correction permet de comprendre l'emploi de ẃs: il s'agit de rapporter cette idée du finalisme qui ne peut être assumé qu'après l'avoir précisée : oui, nous sommes fin, mais pas l'unique fin, l'homme est "une" fin dans un processus téléologique qui, comme tout processus téléologique, est lui-même duel. ${ }^{21}$ Le renvoi de ce dualisme au dialogue perdu Sur la Philosophie ${ }^{22}$ n'est, malheureusement, d'aucun secours. ${ }^{23}$

\section{${ }^{20}$ Cf. Phys II, 8, 199a 8-20 ; II, 2, 194a 21; Voir aussi Protr. fragment 11.}

${ }^{21}$ Il est à noter que l'accent est mis sur le processus et la chose même en transformation, plutôt que sur ses destinataires comme dans la téléologie anthropocentrique ou providentialiste.

${ }^{22}$ Sur De la Philosophie, voir J. Bernays, Die Dialoge des Aristoteles in ihrem Verhältnisse zu seinen übrigen Werken, Berlin, W. Hertz, 1863, p.107-110; Voir aussi: M. Untersteiner, Della filosofia ; introduzione, testo, traduzione e commento esegetico di Mario Untersteiner, Roma, Edizioni di Storia e letteratura, 1963, pp. 56 et 284-285.

${ }^{23}$ Les autres passages dans lesquels Aristote parle explicitement de dualité de la fin sont: DA II, 4, 415b 2 et 20 ; EE VIII, 3, 1249b 15. Ce thème n'a pas attiré beaucoup l'attention des commentateurs. Il a été remis à l'ordre du jour plus récemment, dans les études concernant la 
Mais, à s'en tenir à logique du processus technique d'après notre passage, il n'est pas difficille de discerner ces deux fins, il y a, d'une part, celle supposée: le produit réalisé, ou sa nouvelle forme ; d'autre part, celle qu'Aristote veut ici avancer: nous qui usons de ces produits. Si l'on revient aux exemples cités plus haut, il s'agit d'un côté, d'une fin qui correspond produits eux-mêmes, à savoir la laine ou la navette, de l'autre, la fin qui est l'usager, c'est-à-dire le tisserand.

Interrogeons-nous à présent sur le sens de cette argumentation pour la question de la dépendance de la matière à l'égard de la fin. La suite du texte nous l'indique

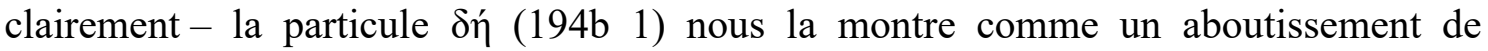

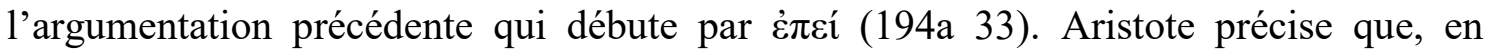
conséquence de ce qui a été dit - à savoir que l'usager est aussi une fin -, même la

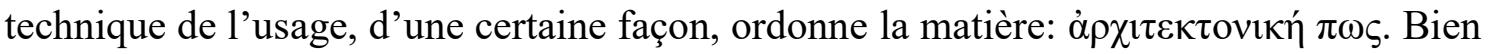
évidemment, l'ordonnatrice au sens propre, c'est plutôt la technique qui commande la production, puisqu'elle a une prise directe sur la matière. Mais, comme nous venons de le voir dans la première distinction de la production $\alpha \pi \lambda \tilde{\omega} \varsigma$ et $\varepsilon v ̉ \varepsilon \rho \gamma o ́ v$, il n'y a pas de technique productrice sans l'orientation antérieure de l'usager qui dicte la nouvelle forme. Celle-ci, en effet, n'est pas déterminée par le hasard ou par une quelconque forme en soi, mais plutôt par une fonction ou un usage spécifique.

Nous arrivons ainsi au point central où Aristote veut en venir dans ces considérations méthodologiques de philosophie naturelle: montrer la dépendance de la matière à l'egard de la fin, non pas en tant qu'il s'agit d'un destinataire, comme l' homme, mais plutôt en tant que celui-ci est un usager, ou encore, la fin en tant que la chose ellemême est mise en usage, ou bien qu'elle accomplit une fonction. ${ }^{24}$ Par analogie avec la

finalité à propos du Premier Moteur dans Meta $\Lambda, 7,1072$ b 2-3. Sur cela, on peut consulter l'étude de F. Baghdassarian: "Les deux sens de la cause finale chez Aristote et le cas des êtres immobiles", version en ligne: http://aitia.hypotheses.org/publications/textes. D'autres études traitent ex professo du thème comme celle de K. Gaiser : "Das zweifache Telos bei Aristoteles", in Naturphilosophie bei Aristoteles und Theophrast, 4th Symposium Aristotelicum, éd. I. Düring, Heidelberg, L. Stiehm, 1969, pp. 97-113. Plus récemment, M.R. Johnson note son importance: Aristotle on Teleology, New York, Oxford University Press, 2005, pp. 64-80. Voir aussi l'intéressante étude de T. K. Johansen, "Two kinds of telos in Aristotle: The view from the De Anima”, in David Ebrey (ed.) Theory and Practice in Aristotle's Natural Science, Cambridge University Press, 2015, pp. 119-136.

${ }^{24}$ Notons que O. Hamelin avait déjà remarqué que c'est la notion d'usage qui domine l'argumentation de ce passage de Physique II. Cf. Aristote, Physique II. Traduction et commentaire par O. Hamelin, Paris, J. Vrin, 1972, p. 71. L'usage a, par conséquent, un sens précis, et ne relève pas du pur arbitraire humain. Sur l'idée de l'usage comme finalité, on peut 
technique, en effet, il montre que la matière n'est pas seulement dépendante de la production, ce qui serait une pure cause efficiente, mais elle l'est également de l'usager, même si celui-ci lui est postérieur et lui semblerait soumis. Son exemple dans la suite, en

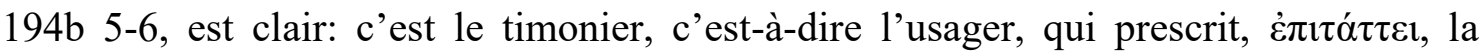
production du gouvernail que le constructeur naval demande à son producteur, le menuisier, qui la réalisera. Ainsi, c'est bien la fin dans l'usage qui détermine la production, et, par conséquent, la matière. Autrement dit, la production du menuisier n'est pas seulement en vue d'obtenir un artefact, car les choses artificielles ne sont ce qu'elles sont que dans la mesure où elles sont adaptées à l'utilisateur, et où elles peuvent accomplir une fonction qui leur est assignée dans un usage propre.

Aristote avance ainsi une idée assez présente, et même fondamentale, dans ses traités biologiques - analogiquement bien entendu, sans l'extériorité du processus technique. Nous l'avons déjà évoquée dans le cas de la constitution de la langue dans le texte cité ci-dessus, et il est possible de la voir encore, quand il écrit par exemple dans Parties des Animaux que "la nature fait des organes pour la fonction, et non pas la fonction pour les organes". ${ }^{25}$ Pareillement, dans l'analogie technique dans Physique II, c'est l'usage qui détermine la production et jamais l'inverse. En effet, ce n'est pas le gouvernail - la matière pour l'usager - qui crée la navigation, mais c'est celle-ci, c'est-à-dire la fonction, qui, à travers le navigateur, prescrit la production du gouvernail. ${ }^{26}$

Enfin, une affirmation à la fin du passage de Physique II que nous étudions, aux lignes 194b 7-8, nous permet de mieux comprendre l'intérêt du philosophe de la nature dans la première distinction des techniques, celle entre les productions $\dot{\alpha} \pi \lambda \tilde{\omega} \varsigma$ et $\varepsilon v ̉ \varepsilon \rho \gamma o ́ v$. Aristote déclare qu'à la différence de la technique où la matière est produite par le travailleur, dans la nature, la matière n'est pas produite, elle est supposée. On peut comprendre par là que l'analogie entre technique et nature doit être considérée avant tout dans le cas de la production củepyóv, c'est-à-dire dans l'adaptation d'une matière à une

voir : Meta $\Theta, 8,1050$ a 23-28; ou encore sur l'identité de la notion d'usage, $\chi \rho \tilde{\eta} \sigma 1 \varsigma$, et celle de fonction, है $p \gamma o v$ : $E E$ II, 1, 1218b 35 - 1219a 1.

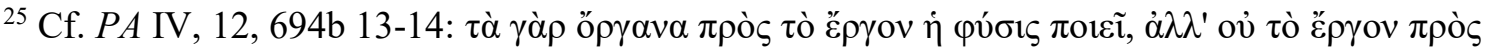
$\tau \grave{\alpha}$ ö $\rho \gamma \alpha v \alpha$.

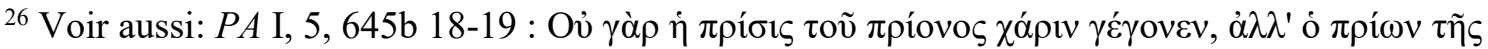

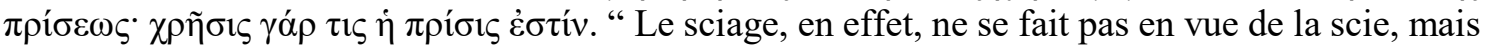
la scie en vue du sciage, car le sciage est une certaine utilisation de la scie" (trad. P. Pellegrin, Aristote, Euvres Complètes, cit., p. 1433). 
fonction, ainsi que dans la technique de l'usage, plutôt que dans celle de la production $\dot{\alpha} \pi \lambda \tilde{\omega} \varsigma_{.}{ }^{27}$

Il est clair, d'après ces précisions, que le but d'Aristote dans ce passage de Physique II, 2, 194a 27 - 194b 9 est d'éclairer ses analyses causales dans les processus naturels en montrant la dépendance de la matière à l'égard de la fin par un raffinement du sens de la téléologie. Le philosophe de la nature ne peut, en effet, se limiter à considérer seulement les composantes matérielles de la réalité, il doit aussi considérer sa forme, et celle-ci non pas en soi, mais dans sa mise en usage qui lui donne sa signification. Plus qu'un exposé comparatif entre des connaissances techniques et naturelles, ces lignes de Physique II nous montrent un processus téléologique duel grâce auquel la réalité, dans son devenir artificiel ou naturel, n'est pas limitée à ce qu'on a appelé sa finalité interne, c'est-à-dire sa forme, mais, grâce à la notion d'usage comme fin, elle est inscrite dans une dynamique, en rapport à d'autres réalités ou à une totalité donnée, là où sa forme est en usage.

L'usage et la fin $\tau o ̀ ~ \hat{\omega}$

Enfin, nous voudrions noter une confirmation de notre interprétation de ce passage de Physique II par la lecture d'un autre texte dans lequel il est aussi question de dualité de la fin : De Anima II, 2, 415b 15-21, où Aristote traite du problème du rapport entre âme et corps dans la perspective de la causalité. C'est après avoir considéré l'âme comme forme du corps vivant, qu'il traite de la causalité finale, et qu'il précise brièvement le sens

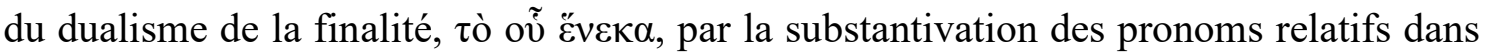
la formule $\tau$ ò oũ et $\tau$ ò $\tilde{\omega}:$

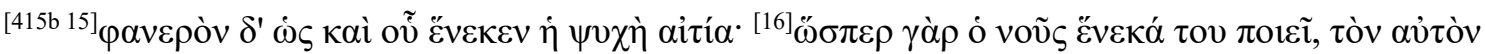

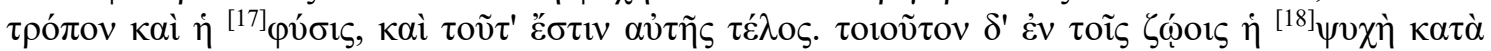

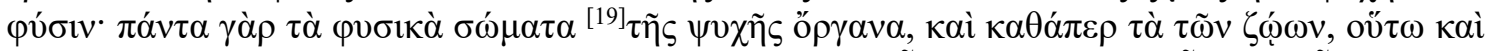

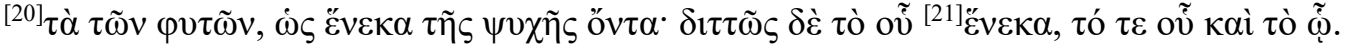

Il est clair, d'un autre côté, qu'à titre de fin, l'âme est également cause poursuivie. De même, en effet, que l'intelligence poursuit une fin dans ce qu'elle fait, de la même manière la nature. Et c'est ce qui constitue son but. Or, chez les animaux, c'est l'âme qui joue naturellement ce genre de rôle. Car tous les corps naturels constituent des instruments de l'âme, et ce qui est vrai pour les corps des animaux l'est aussi pour ceux des végétaux, au sens où ils existent en vue de l'âme.

${ }^{27}$ Voir les analogies avec les techniques d'usage, par exemple, dans $P A$ III, 2, 663b 21- 25 ; GA II, 6, 744b 11-26. 
Et la fin est double : le but visé et le sujet servi. (Trad. R. Bodéüs, modifiée : Aristote, Euvres Complètes, cit., p. 996).

Afin de justifier la causalité de l'âme comme fin du corps, Aristote l'inscrit dans une vue plus fondamentale : celle de l'orientation téléologique dans la nature elle-même. Celle-ci, à son tour, est expliquée à travers l'analogie de l'intelligence pratique qui produit en vue de quelque chose, ò voṽ $\varepsilon \check{v \varepsilon \kappa a ́ ~ \tau o v ~ \pi o r \varepsilon i ̃ . ~ I l ~ a f f i r m e ~ a l o r s ~ d ' e m b l e ́ e ~ s a ~ t h e ̀ s e: ~ l ' a ̂ m e ~}$ est cause finale et les corps naturels sont ses instruments, ö $\rho \gamma \alpha v \alpha$, car ils sont en vue des fonctions vitales dont l'âme est le principe.

Tout comme dans notre passage de Physique II, c'est aussi dans l'exposition de cette idée de fin dans l'usage - non plus par l'homme mais par l'âme - qu'Aristote

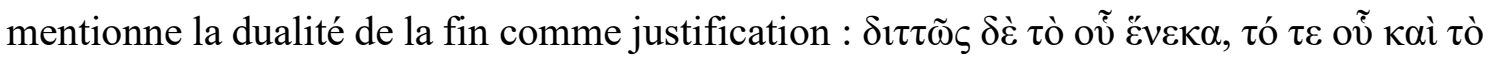
$\tilde{\omega}$. Sans pouvoir entrer ici dans la discussion du sens de cette brève formule, notons seulement que le deuxième terme, la fin $\tau$ ò $\tilde{\omega}$, par l'idée de destinataire qu'évoque le datif, indique qu'il s'agit là assez vraisemblablement du sens de l'âme comme fin, c'est-à-dire en tant qu'elle se sert du corps, celui-ci étant pris dans son aspect organique, fonctionnel. ${ }^{28}$ L'âme est fin en tant qu'elle permet l'usage, au sens de la réalisation du corps par ses fonctions vitales. C'est en cela que l'âme comme destinataire, fin $\tau$ ò $\tilde{\hat{\omega}}$, est pour le corps un bien et, donc, une fin au sens propre. La grande différence entre les processus technique et les processus naturelle est l'immanence de ce dernier: l'âme n'est pas externe au corps vivant, comme c'est le cas de l'homme pour l'artefact. Dans les deux cas, néanmoins, Aristote prend soin de justifier cette destination téléologique en montrant

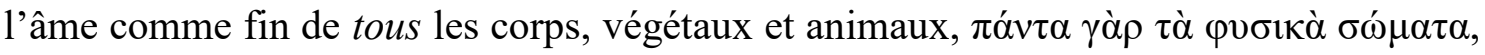
ainsi que dans Physique II où l'homme est considéré comme fin de toutes choses. ${ }^{29}$

L'autre sens de la fin, tò oũ, pour le corps, si on lit ce texte en parallèle avec Physique II, ce serait celui du corps comme matière vivante, le composé hylémorphique, l'analogue pour la technique de la fin en tant que produit constitué. Tout comme l'artefact (fin $\tau$ ò oũ), le corps vivant n'a pas non plus de sens sans sa mise en usage en des fonctions

${ }^{28}$ O. Hamelin note la parenté entre l'idée d'usage dans Physique II et celle de fin $\tau$ ò $\tilde{\omega}$ du $D e$ Anima. Cf. Aristote, Physique II, cit., p. 71.

${ }^{29}$ Comme on a parlé d'une téléologie anthropo-centrique dans Physique II, ne faudrait-il pas alors parler ici d'une téléologie psycho-centrique? Ou ne serait-il pas plus juste de voir l'élément commun aux deux passages du De Anima et de Physique et parler plutôt d'une téléologie ergocentrique? 
spécifiques (fin $\tau$ ò $\tilde{\omega})$. En effet, "toutes choses se déterminent par leur fonction" ${ }^{30}$, et, de ce fait, la finalité ne peut être que double.

Nélio Gilberto Dos Santos

Doctorant Paris IV - Sorbonne

\section{Bibliographie}

Aristote, Euvres Complètes, sous la direction de P. Pellegrin, Paris, Flammarion, 2014.

Aristote, The complete works of Aristotle: the revised Oxford translation, Vol. I, Princeton, N.J, Princeton University Press, 1984.

Aristote, Aristoteles Latinus, VII. 1. 2, Physica, Leiden; New York, E. J. Brill, 1990.

Aristote, Physique II, traduction et commentaire par O. Hamelin, Paris, J. Vrin, 1972 (1907).

Aristote, La Physique, texte établi et traduit par H. Carteron, 2 vol., Paris, Les Belles Lettres, 1990 (1926-1931).

Aristote, Aristotle's Physics, a revised text with introduction and commentary by W. D. Ross, 2 vol., Oxford, Clarendon, 1936.

Bachdassarian, F., " Les deux sens de la cause finale chez Aristote et le cas des êtres immobiles " [en ligne], http://aitia.hypotheses.org/publications/textes (Page consultée 26 juillet 2017).

Gaiser, K., " Das zweifache Telos bei Aristoteles ”, in Naturphilosophie bei Aristoteles und Theophrast, 4th Symposium Aristotelicum, éd. I. Düring, Heidelberg, L. Stiehm, 1969, pp. 97113.

Johansen, T. K., "Two kinds of telos in Aristotle : The view from the De Anima", in David Ebrey (ed.) Theory and Practice in Aristotle's Natural Science, Cambridge University Press, 2015, pp. 119-136.

Johnson, M. R., Aristotle on Teleology, New York, Oxford University Press, 2005.

Leunissen, M., Explanation and teleology in Aristotle's science of nature, New York, Cambridge University Press, 2010.

Lerner, M-P., Recherches sur la notion de finalité chez Aristote, Paris, PUF, 1969.

Mansion, S., " Un passage obscur du deuxième livre de la Physique (ch. 2, 194a27-b8) ", in Kephalaion, Studies in Greek philosophy and its continuation offered to C. J. de Vogel, éds. by J. Mansfeld \& L.M. de Rijk, Assen, van Gorcum, 1975, pp. 70-77. Repris dans S. Mansion, Études Aristotéliciennes, recueil d'articles, avant-propos, bibliographie, indices de J. Follon, Louvain-laNeuve, Ed. de l'Institut supérieur de philosophie, 1984.

Sedley, D., “Is Aristotle's Teleology Anthropocentric?”, Phronesis, 1991, no 36, pp. 179-197.

Sedley, D., "Teleology, Aristotelian and Platonic", in G. Lennox et R. Bolton (éd.), Being, Nature and Life in Aristotle. Essays in Honor of A. Gotthelf, Cambridge, Cambridge University Press, 2010, 5-29.

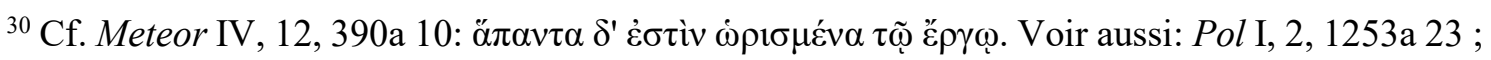
Cae 286a 8-9. 